\title{
Direct Evidence of Reduction of Cloud Water after Spreading Diatomite Particles in Stratus Clouds in Beijing, China
}

\author{
Qiang Zhang, ${ }^{1}$ Mengyu Huang, ${ }^{1,2}$ Xuexi Tie, ${ }^{3}$ and Xincheng $\mathrm{Ma}^{1}$ \\ ${ }^{1}$ Beijing Weather Modification Office, Beijing, China \\ ${ }^{2}$ Department of Atmospheric Science, School of Physics, Peking University, Beijing, China \\ ${ }^{3}$ National Center for Atmospheric Research, Boulder, CO 80303, USA \\ Correspondence should be addressed to Mengyu Huang, mengyu2324@yahoo.com.cn \\ Received 22 August 2009; Revised 27 January 2010; Accepted 20 February 2010 \\ Academic Editor: Shouting Gao
}

Copyright ( $) 2010$ Qiang Zhang et al. This is an open access article distributed under the Creative Commons Attribution License, which permits unrestricted use, distribution, and reproduction in any medium, provided the original work is properly cited.

\begin{abstract}
Artificial weather modification experiments have been intensively practiced in many years over China, and some progresses have been made, including more methodologies and advanced instruments. However, a challenge question still remains for providing convincing scientific evidence during these practices and experiments. This is a very difficult scientific issue, which is related to complicated cloud physical science, such as to accurately predict the large natural variability of cloud formation and precipitation. In this study, we report a clear evidence that the cloud water is reduced after spreading diatomite particles in stratus clouds during a field experiment in Beijing, China. The analysis shows that the diatomite particles (15-20 $\mu \mathrm{m}$ in radius) are large and have strong hygroscopic property (absorbing cloud water). As a result, during the experiment, spreading large diatomite particles lead to downward motion (producing more stable atmospheric condition) and reduction of cloud water. It is noted that due to lacks of instruments, this designed experiment only can provide a qualitative result (such as photo evidence), and no quantitative result can be drawn from this experiment.
\end{abstract}

\section{Introduction and Methods}

The theory and experiment for artificial weather modifications have a long history. The artificial weather modifications include to enhance or to reduce cloud formation and precipitation. The early experiments for artificial reduction of precipitation occurred in early 1940s. For example, Langmuir and his colleagues discovered that silver iodide (AgI) had nucleating ability to cause a rapid conversion of the water to ice, leading quickly to the production of snowflakes and the dissipation of cloud in the seeded region. Since then, AgI is widely used in cloud seeding process. In a general case, increase in numbers of cloud condensation nuclei (CCN) leads to enhancement of cloud formation and precipitation. However, if the numbers of $\mathrm{CCN}$ exceed a certain amount in clouds (overspread), with a limited amount of water contents available in clouds, a large amount of small cloud particles is formed to inhibit the formation of large rain droplets. Instead of enhancement of precipitation, the overspreading AgI particle is often considered as artificial reduction of precipitation. Li et al. [1] used a state-of-theart dynamical model, Weather Real time Forecast (WRF), coupled with a detailed cloud micro-physical model to quantify the relationship between CCN and precipitation. Their result illustrated that there is a threshold CCN value for whether the increase in CCN causes an enhancement or deduction for precipitation (see Figure 1). The threshold value is varied with different cloud conditions and ranges from 2000 to $5000 \# / \mathrm{cm}^{3}$. This model result gives a scientific guidance for either to enhance or to reduce precipitation by artificially adding the nuclei in clouds.

Artificial weather modification experiments have been intensively practiced in many years over China, and some progresses have been made, including more methodologies and advanced instruments. However, a challenge question still remains for providing convincing scientific evidence during these practices and experiments. Because this is a very difficult scientific issue, which is related to complicated cloud physical science, such as to accurately predict the large natural variability of cloud formation and precipitation. 


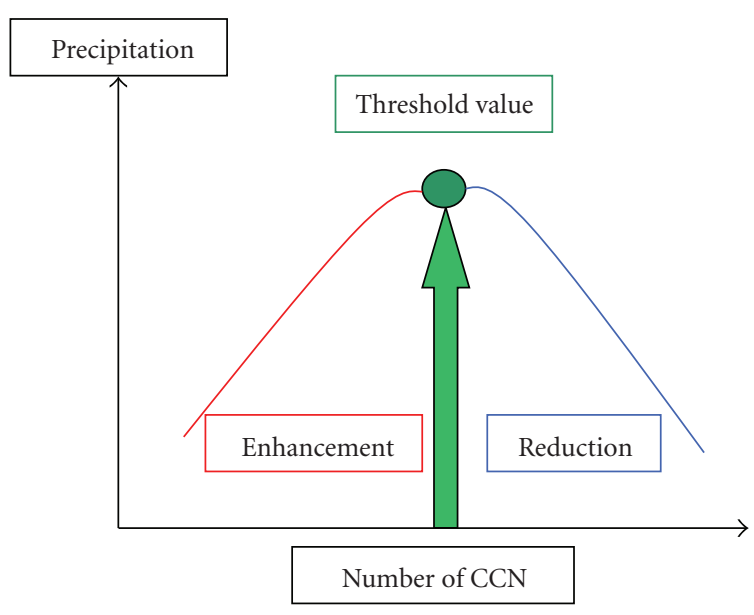

FIGURE 1: Schematic picture for the effect of changes in cloud nuclei $(\mathrm{CCN})$ on precipitation. The red regime represents for the enhancement of precipitation with increase of CCN, and the blue regime represents for the reduction of precipitation with increase of CCN. The green dot indicates the threshold for the enhancement/reduction transition point.

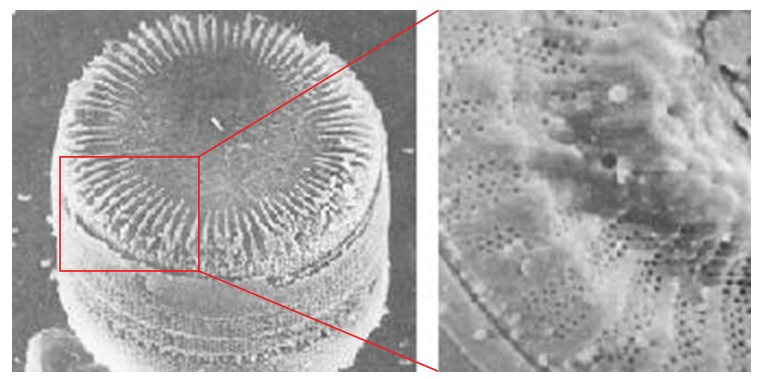

FIgure 2: The typical shape of diatomite particle. The right panel shows a detailed structure of the particle. It clearly shows that there are lot of small holes in the particle, which make the particle with hydrophilic property.

With a large uncertainty in prediction of precipitation, it is difficult to clarify that the amount of cloud water is either enhanced or reduced by artificial weather modification experiments. The progress for this problem is dependent upon an improved fundamental understanding of crucial cloud precipitation and larger-scale atmospheric processes. During the past, there were some successes and indirect evidences that the method for adding nuclei into existing clouds may have important effects on precipitation. For example, Rosenfeld and Farbstein [2] showed that the mineral dust from Sahara Desert provides a large amount of aerosol particles which acted as cloud nuclei. This large amount of particles often produces reduction of precipitation due to the fact that these particles can be considered as overspreading nuclei in clouds. As a result, the limited amount of water content in the clouds formed more small droplets instead of a few large rain droplets, leading to the reduction of precipitation. Zhao et al. [3] showed that the large increases in aerosol particles produce significant reduction of precipitation in Eastern China. Their analysis suggested that the reduction of precipitation induced by the enhancement of aerosol particles is not only due to the cloud nuclei effect, but also the changes in thermodynamical structure of the atmosphere.

In this study, we show another method applied in field experiments for attempting to reduce cloud formation and precipitation. Beijing Weather Modification Office (BWMO) makes several experiments of spreading a large amount of diatomite particles in clouds for affecting cloud formation and precipitation. The major composition of diatomite particles is $\mathrm{SiO}_{2}$, with a small amount of $\mathrm{Fe}_{2} \mathrm{O}_{3}, \mathrm{CaO}$, $\mathrm{MgO}$, and $\mathrm{Al}_{2} \mathrm{O}_{3}$. The averaged radius ranges from 15 to $30 \mu \mathrm{m}$. The detailed structure of diatomite particles is shown in Figure 2. It indicates that diatomite particle has many mini holes on the surface, resulting in a strong hygroscopic property. The diatomite particles have the following effects on the formation of clouds. First, the diatomite particles can easily absorb water vapor acting as cloud nuclei. Second, the diatomite particles are large and heavy, and spreading a large amount of diatomite particles could perturb the vertical motion in clouds. Because upward motion is a key factor for the formation of clouds and precipitation, the downward motion due to spreading a large amount of diatomite particles produces a negative impact on cloud formation. We should, however, mention that due to lacks of instruments, this designed experiment only can provide a qualitative result (such as photo evidence), and no quantitative result can be drawn from this experiment.

\section{Result and Discussion}

The experiment was carefully designed in order to capture clear evidence for the effect on cloud formation after spreading diatomite particles. Two aircrafts were used in the experiment. One aircraft (An-26) was used to spread diatomite particles, and another aircraft (XY-II) was used to take pictures during the experiment. Ideally, quantitative cloud water reduction should be measured after the experiment, which can provide important information for the experiment result. However, this requires a very complicated experiment design and needs at least 3 airplanes to achieve the goal. In this experiment, only 2 airplanes were available and used. One was used for seeding and another was used for taking photos. There was no extra airplane to be used to measure the cloud physical properties before and after the seeding. As a result, the designed experiment only can provide a qualitative result (such as photo evidence), but no quantitative result can be drawn from this experiment.

Figure 3 shows evidence that after the spreading of diatomite particles, there was a clear cloud breaking area along the spreading route. This particular flight route was taken on September 19, 2005 from 17:04 to 17:09 pm with 5 -minute period. The speed of aircraft was about $300 \mathrm{~km} / \mathrm{hr}$, and the flight height was at $4.5 \mathrm{~km}$ during the spreading time. The picture was taken by the XY_II aircraft at $17: 12$ pm after 3 minutes of the spreading at the flight height of $6.0 \mathrm{~km}$. According to the above information, the length of the cloud breaking area is estimated to be $25 \mathrm{~km}(5 \mathrm{~km} / \mathrm{min} \times 5 \mathrm{~min})$, and the width of the area is estimated to be less than $1 \mathrm{~km}$ 


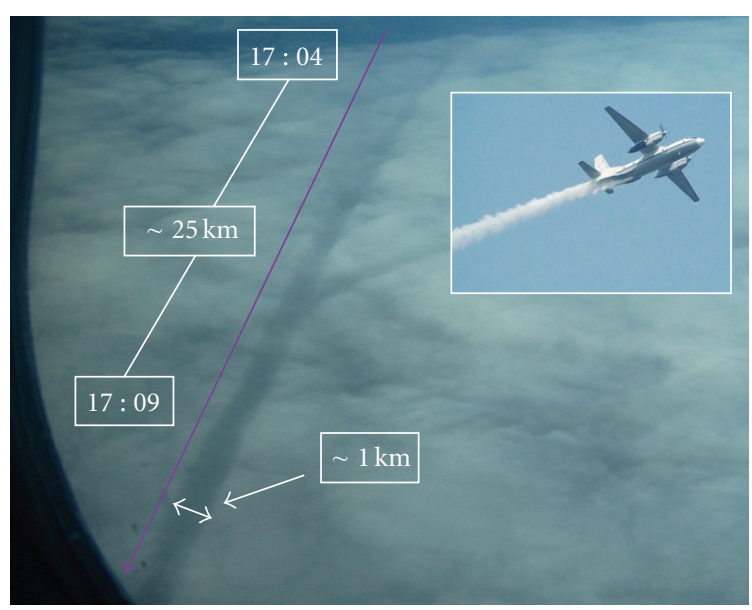

FIgUre 3: The photo is taken by the aircraft (XY-II) after the spreading. It shows a direct evidence that after the spreading of diatomite particles, there is clearly a cloud breaking region along the spreading route. The flight was taken on September 19, 2005 from 17:04 to $17: 09$ pm with 5 minutes. The flight speed is about $300 \mathrm{~km} / \mathrm{hr}$, and flight height of $4.5 \mathrm{~km}$ during spreading time. The estimated length and width are approximately 25 and less than $1 \mathrm{~km}$, respectively. The right-upper panel illustrates the spreading of particles.

by assuming that the width is smaller than $1 / 25$ of the length (see Figure 3). The microphysical property of the cloud was also measured before the spreading (shown in Figure 4). The measurement suggests that the cloud layer was centered at $3.4 \mathrm{~km}$, and thickness of cloud layer was about 200-300 meters. The cloud water content was about $0.04 \mathrm{~g} / \mathrm{m}^{3}$ and the number concentrations of cloud particles ranged from 120 to $160 \# / \mathrm{cm}^{3}$ with mean effective diameter of $8.5-9.5 \mu \mathrm{m}$. These measured data suggests that this was a relatively thin stratus cloud with a medium amount of cloud water.

The causes for the reductions of cloud water by the spreading diatomite particles are analyzed. The aircraft used for the experiment was the An-26 airplane with a spreading rate $(13.3 \mathrm{Kg} / \mathrm{km})$ for diatomite particles during the experiment. In a 5 -minute period, the total weight of 332 $\mathrm{kg}$ diatomite particles was spread in this breaking area. The total number of diatomite particles $\left(N_{T}\right)$ can be estimated as follows:

$$
N_{T}=W E I_{T} / W E I_{S},
$$

where $\mathrm{WEI}_{\mathrm{T}}$ represents the total weight of diatomite (332 $\mathrm{Kg}$ ) and $\mathrm{WEI}_{\mathrm{S}}$ represents the weight for a single particle of diatomite, which can be estimated by

$$
W E I_{S}=\frac{4}{3} \pi r^{3} \rho_{s},
$$

where $\rho_{\mathrm{s}}$ represents the density of a single diatomite particle $\left(0.5 \mathrm{~g} / \mathrm{cm}^{3}\right)$, and $r$ is the mean radius of diatomite particles $(\sim 20 \mu \mathrm{m})$. As a result, a single particle of diatomite weight (WEI $I_{S}$ ) is equal to $1.7 \times 10^{-8} \mathrm{~g}$, and the $N_{T}$ is equal to $1.9 \times 10^{14}$. The total area $\left(A_{T}\right)$ of the cloud breaking area (with $25 \mathrm{~km}$ in the length and $1 \mathrm{~km}$ in the width) is estimated to be $2.5 \times 10^{11} \mathrm{~cm}^{2}$, and the area density of diatomite

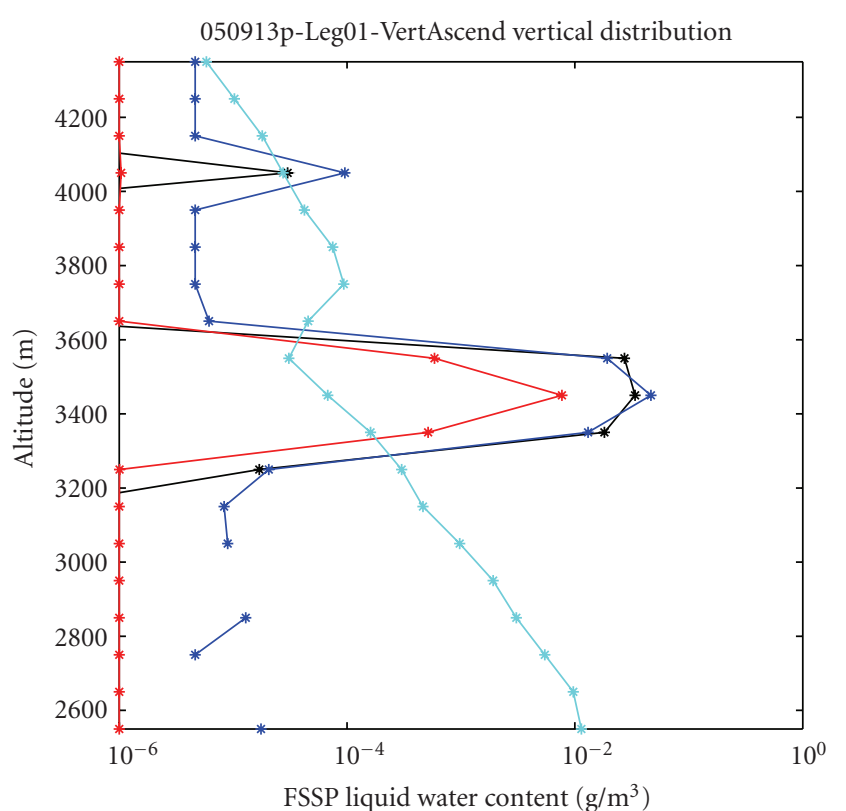

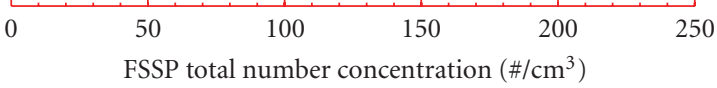

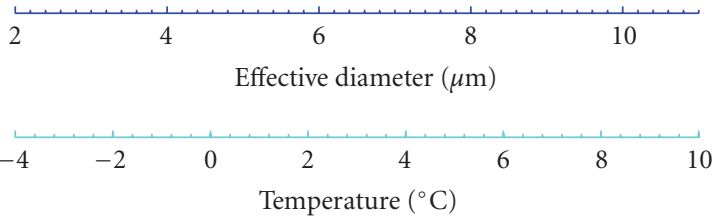

FIGURE 4: Measured vertical distribution of microphysical property of the cloud before the spreading. The result suggests that the cloud layer is centered at $3.4 \mathrm{~km}$, and thickness of cloud layer is about 200300 meters. The cloud water content is about $0.04 \mathrm{~g} / \mathrm{cm}^{3}$ and the number of cloud particles ranges from 120 to $160 \# / \mathrm{cm}^{3}$ with mean effective diameter of $8.5-9.5 \mu \mathrm{m}$.

particles spread in the cloud is estimated $\left(N_{T} / A_{T}\right)$ to be $760 \# / \mathrm{cm}^{2}$. The terminal velocity of the particles is about $313 \mathrm{~m} / \mathrm{min}$. With the cloud thickness of $200-300 \mathrm{~m}$, the particles take less than 1 minute to penetrate the cloud layer. As a result, the large quantity of heavy particles produces strong perturbations on the organized upward motion (required for cloud formation). In addition, because the diatomite particles have strong hygroscopic property, they absorb cloud water during sedimentation in the cloud layer. Both the effects produce unfavorable conditions for the cloud formation and result in the reduction of cloud water. However, the impact on clouds during this experiment is moderate in the breaking area. In order to enhance the impact on clouds by spreading diatomite particles, a large payload aircraft is needed in future experiments.

\section{Summary}

In this study, we report a clear evidence that cloud water was reduced after spreading diatomite particles in stratus clouds during an experiment in Beijing, China. The analysis 
of the experiment result suggests that diatomite particles have strong hygroscopic property, and the particles strongly absorb cloud water. Furthermore, the diatomite particles are very large with radius of $15-20 \mu \mathrm{m}$. As a result, the gravity sedimentation of the diatomite particles produces strong downward motion, leading to atmosphere in more stable conditions. Both the effects intend to reduce cloud water in the existing clouds. However, the result of this experiment was moderate. More experiments should be conducted and more evidences should be collected. A large payload airplane should be used in future experiments in order to produce more effective effects in this kind experiment. We should, however, mention that due to lacks of instruments, this designed experiment only can provide a qualitative result (such as photo evidence), and no quantitative result can be drawn from this experiment.

\section{Acknowledgments}

This research is partially supported by Science and Technology Administration of China under Grant no. 2006BAC12B00, China Meteorological Administration (CMA) under Grant no. GYHY(QX)2007-6-36, National Basic Research Program of China (2006CB403701), Beijing Excellent Talent Fundation under Grant no. 20071D020800058, and National Natural Science Foundation of China (NSFC) under Grant no. 40575060 and 40705046. The National Center for Atmospheric Research is sponsored by the National Science Foundation and operated by UCAR.

\section{References}

[1] G. Li, Y. Wang, and R. Zhang, "Implementation of a twomoment bulk microphysics scheme to the WRF model to investigate aerosol-cloud interaction," Journal of Geophysical Research D, vol. 113, no. 15, Article ID D15211, 2008.

[2] D. Rosenfeld and H. Farbstein, "Possible influence of desert dust on seedability of clouds in Israel," Journal of Applied Meteorology, vol. 31, no. 7, pp. 722-731, 1992.

[3] C. Zhao, X. Tie, and Y. Lin, "A possible positive feedback of reduction of precipitation and increase in aerosols over eastern central China," Geophysical Research Letters, vol. 33, no. 11, Article ID L11814, 2006. 

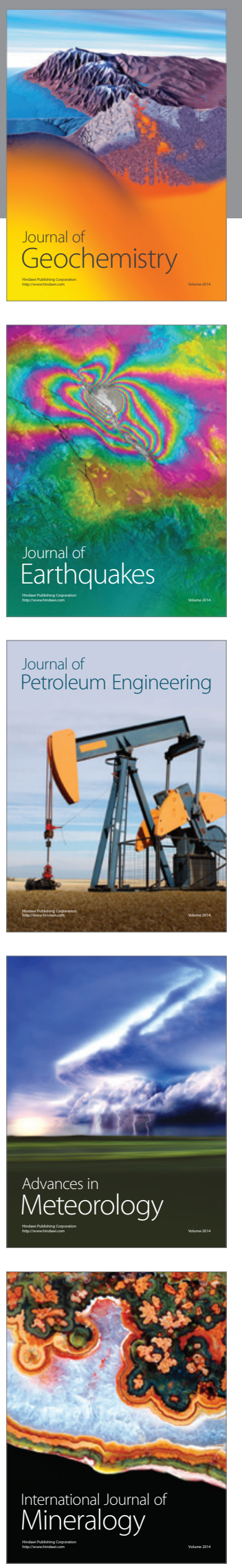
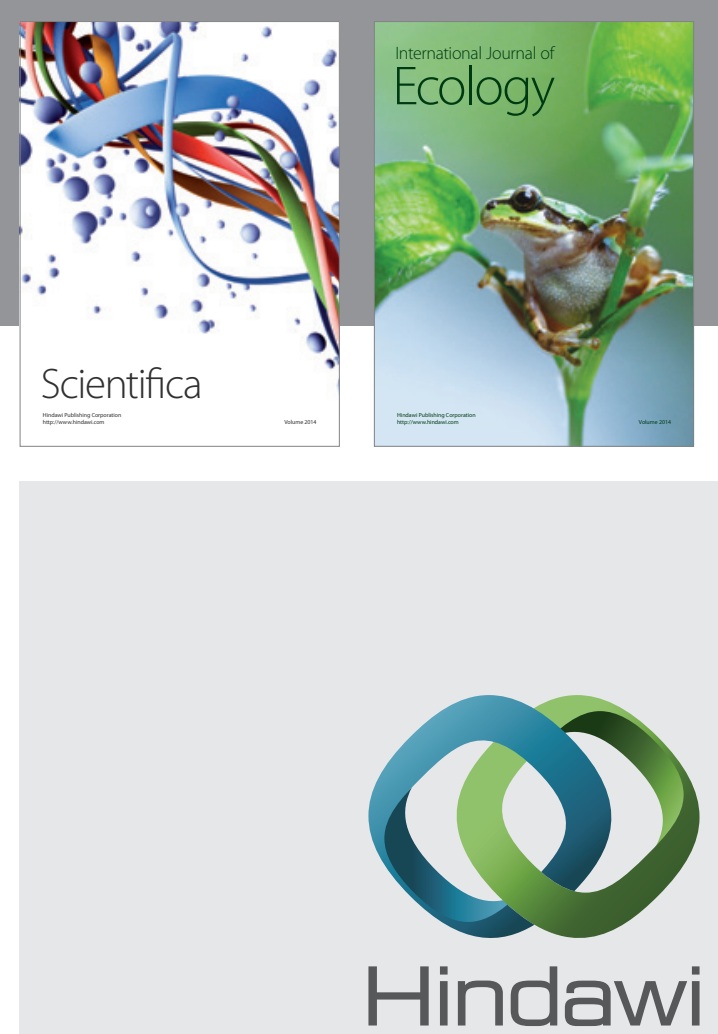

Submit your manuscripts at http://www.hindawi.com
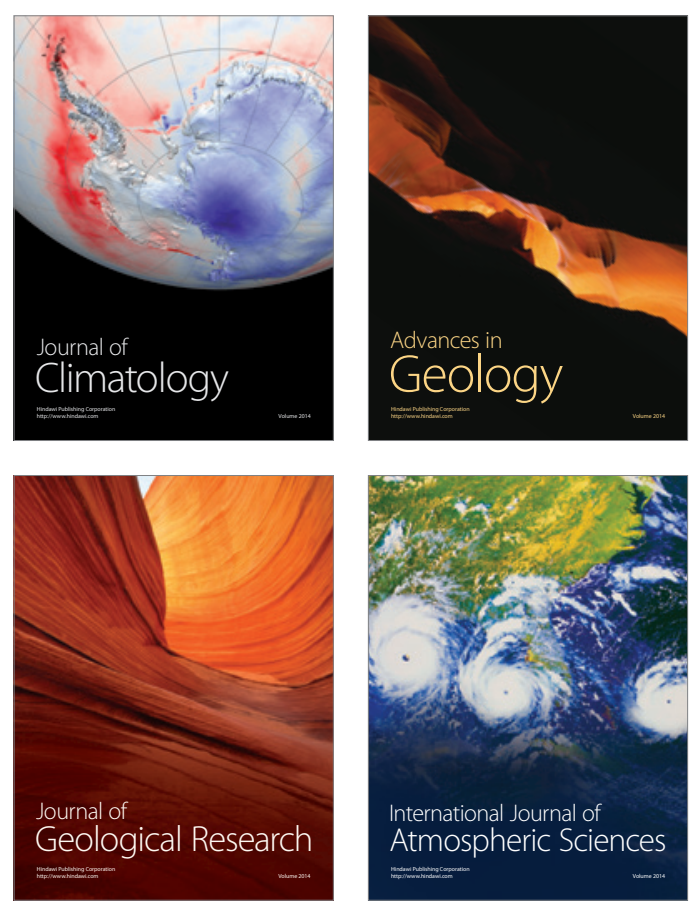
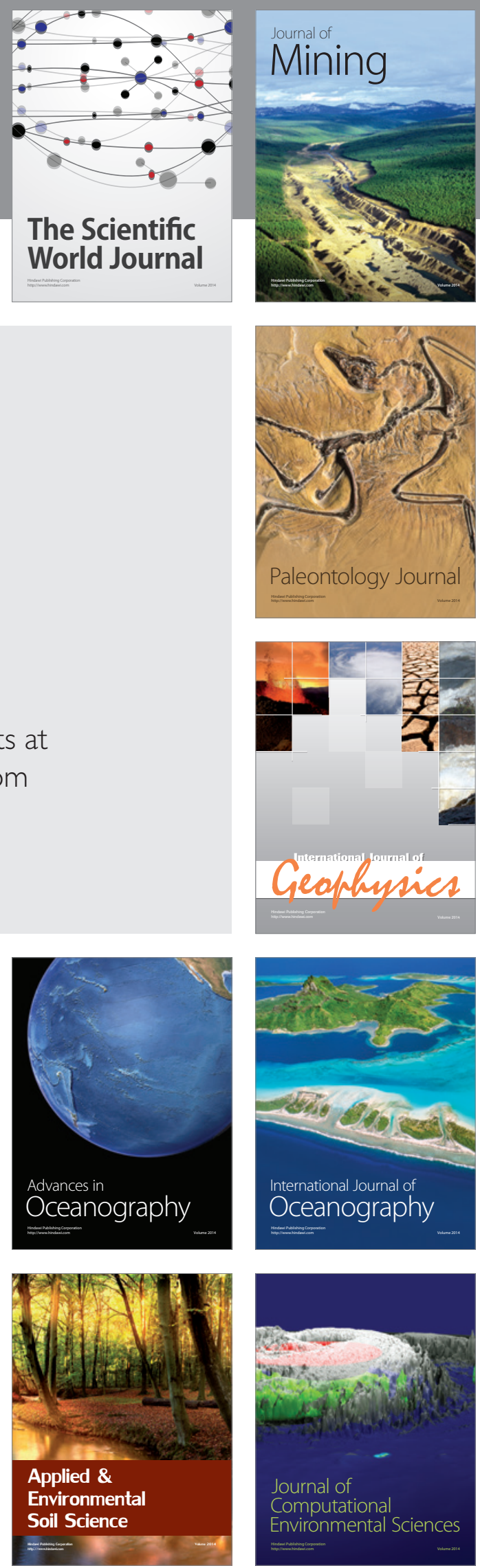